\title{
Autograft vs Allograft Comparison in Pediatric Medial Patellofemoral Ligament Reconstruction
}

\author{
Tariq Hendawi, MD, ${ }^{1}$ Brian Godshaw, MD, ${ }^{1,2}$ Christopher Flowers, MD, ${ }^{1}$ Isabel Stephens, MD, ${ }^{1,2}$ \\ Lawrence Haber, MD, ${ }^{1,2}$ Sean Waldron, $M^{1,2}$ \\ ${ }^{1}$ Department of Orthopedic Surgery, Ochsner Clinic Foundation, New Orleans, LA ${ }^{2}$ The University of Queensland Faculty of Medicine, \\ Ochsner Clinical School, New Orleans, LA
}

Background: Patella instability and medial patellofemoral ligament (MPFL) injury are frequently encountered in pediatric patients. MPFL reconstruction is often chosen to treat this condition with good results; however, no consensus has been reached about which graft or technique to use. The purpose of this study was to evaluate the differences in graft survivorship, clinical outcomes (assessed with Kujala scores), and cost between autograft and allograft usage in MPFL reconstruction in pediatric patients.

Methods: In this retrospective review of patients who underwent MPFL reconstruction between 2012-2015, autograft gracilis tendon was used for Group 1, and allograft gracilis tendon was used for Group 2. Outcomes were graft survivorship, postoperative Kujala scores, operative time, costs, graft size, and tibial tubercle-trochlear groove distance.

Results: Fifty-six patients were included in this study, 21 in Group 1 and 35 in Group 2. No differences in age, sex, or chronicity were seen between the groups. Patients in Group 1 had longer operative times ( 134.5 minutes vs 97.3 minutes, $P=0.0002$ ), higher rates of graft failure $(28.6 \%$ vs $0 \%, P=0.0037)$, and lower Kujala scores ( 80.3 vs $92.1, P=0.0032)$ compared to Group 2 . All graft failures occurred in patients with chronic patella dislocations and occurred an average of 13.8 months postoperatively. Overall, autograft was costlier than allograft because of the cost of reoperation.

Conclusion: This study supports the use of allograft for chronic patellar instability because of improved graft survivorship and clinical outcome scores, as well as the lower cost and reoperation rate.

Keywords: Allografts, autografts, patellar dislocation, patellar ligaments

Address correspondence to Sean Waldron, MD, Department of Orthopedic Surgery, Ochsner Clinic Foundation, 1514 Jefferson Hwy., New Orleans, LA 70121. Tel: (504) 542-3000. Email: sean.waldron@gmail.com

\section{INTRODUCTION}

Patellar instability is a frequently encountered problem in pediatric patients. Nietosvaara et al reported an annual incidence of 43 per 100,000 patellar dislocations in children $<16$ years of age. ${ }^{1}$ Medial patellofemoral ligament (MPFL) injuries and patellar dislocations are most common in females 10-17 years of age. ${ }^{2}$

The MPFL is a thickening of the structures in layer 2 of the knee, running from the superomedial corner of the patella to the medial femoral epicondyle at the Schöttle point. ${ }^{3,4}$ The primary function of the MPFL is to provide medial restraint to the patella and prevent lateral patellar translation or dislocation. Cadaveric studies have demonstrated that the MPFL provides $50 \%-60 \%$ of this medial restraint., Injury to or tearing of the MPFL leads to lateral translation and/or dislocation of the patella. Studies of surgically explored MPFLs in patients with known lateral patellar dislocation have reported MPFL injury in $94 \%-100 \%$ of patients. ${ }^{7,8}$

Initially, nonoperative conservative management of patellar dislocations was the standard of care for MPFL injuries; however, MPFL reconstruction is becoming popular and has good outcomes. ${ }^{9}$ In a randomized controlled trial comparing nonoperative treatment vs MPFL reconstruction, Bitar et al found significantly greater Kujala scores and fewer recurrences/subluxations in the reconstruction group. ${ }^{10}$

Many surgical techniques for MPFL reconstruction have been described, ${ }^{11-19}$ but no single technique has been proven to be significantly superior to the others. The choice of technique may be guided by the complications associated with different techniques. For example, the choice between autograft vs allograft was made in one study because of patient fears of donor morbidity. ${ }^{13}$ Graft fixation techniques - suture fixation or bone tunnel fixation - are associated with their own complications. Bone tunnel fixation has been associated with an overall higher complication rate than suture fixation ( $29.8 \%$ vs $21.6 \%$, respectively); however, suture fixation is associated with higher rates of recurrent instability and hypermobility than bone tunnel fixation (4.8\% vs $3.3 \%$ and $24.0 \%$ vs $8.6 \%$, respectively) ${ }^{20} \mathrm{~A}$ 2015 systemic review by Stupay et al reported an increase in popularity of MPFL reconstruction and satisfactory outcomes despite the use of multiple techniques. ${ }^{21}$ Almost all 


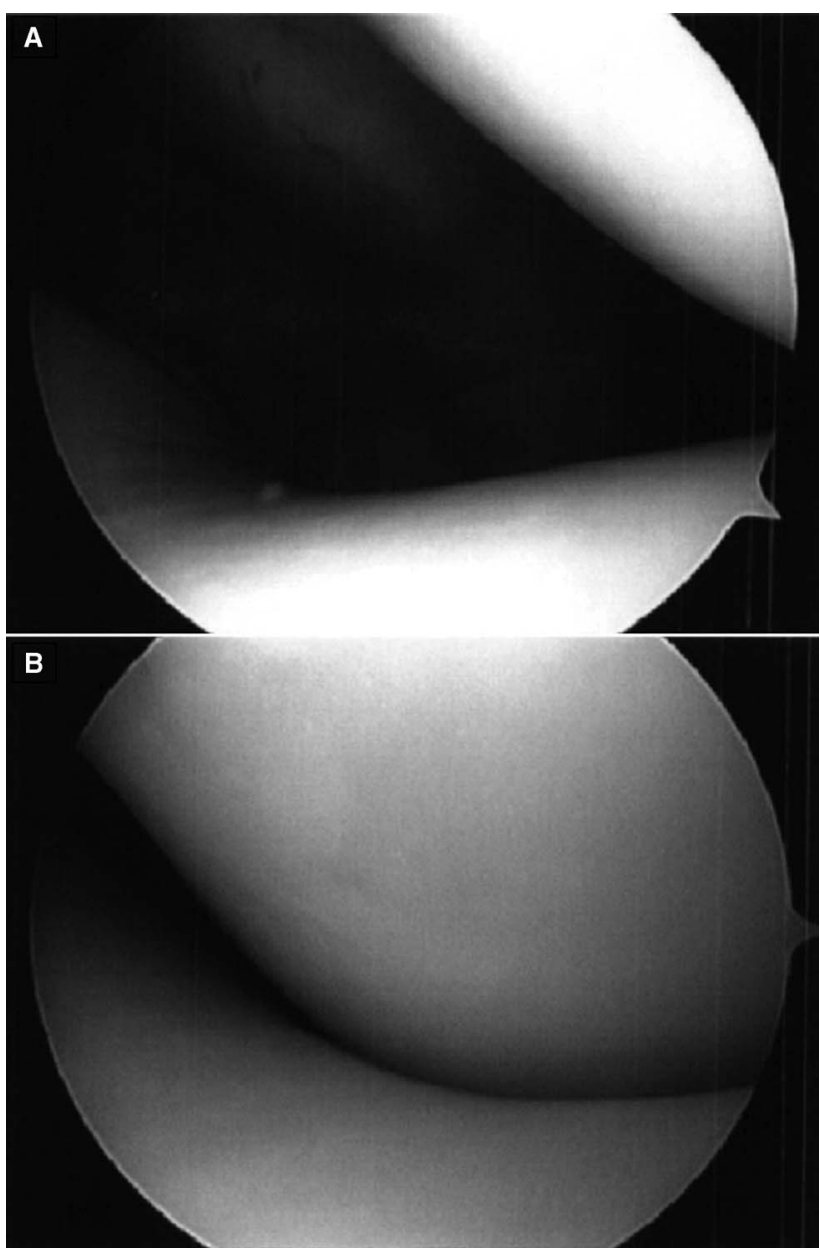

Figure 1. Intraoperative arthroscopic images from superolateral portal demonstrate (A) lateral patella maltracking and (B) appropriate patella tracking after medial patellofemoral ligament reconstruction.

the described techniques used autograft, although allograft is also a viable option.

Despite the abundance of orthopedic literature discussing autograft MPFL reconstruction techniques, the literature is lacking in direct comparisons of autograft vs allograft in pediatric patients. Additionally, the economics of graft choices have yet to be investigated. Direct costs to both the patient and medical centers should be considered in preoperative planning.

The purpose of this study was to directly compare autograft gracilis to allograft gracilis MPFL reconstruction in pediatric patients and explore differences in graft survivorship, postoperative Kujala scores, operative time, and costs. Secondary measures included graft size and tibia tubercletrochlear groove (TT-TG) measurements.

\section{METHODS}

\section{Population}

We conducted a retrospective review of all patients who underwent MPFL reconstruction by a single fellowshiptrained pediatric orthopedic surgeon (S.W.) at our institution from 2012-2015. The patients were divided into 2 cohorts: Group 1 consisted of MPFL reconstructions using gracilis

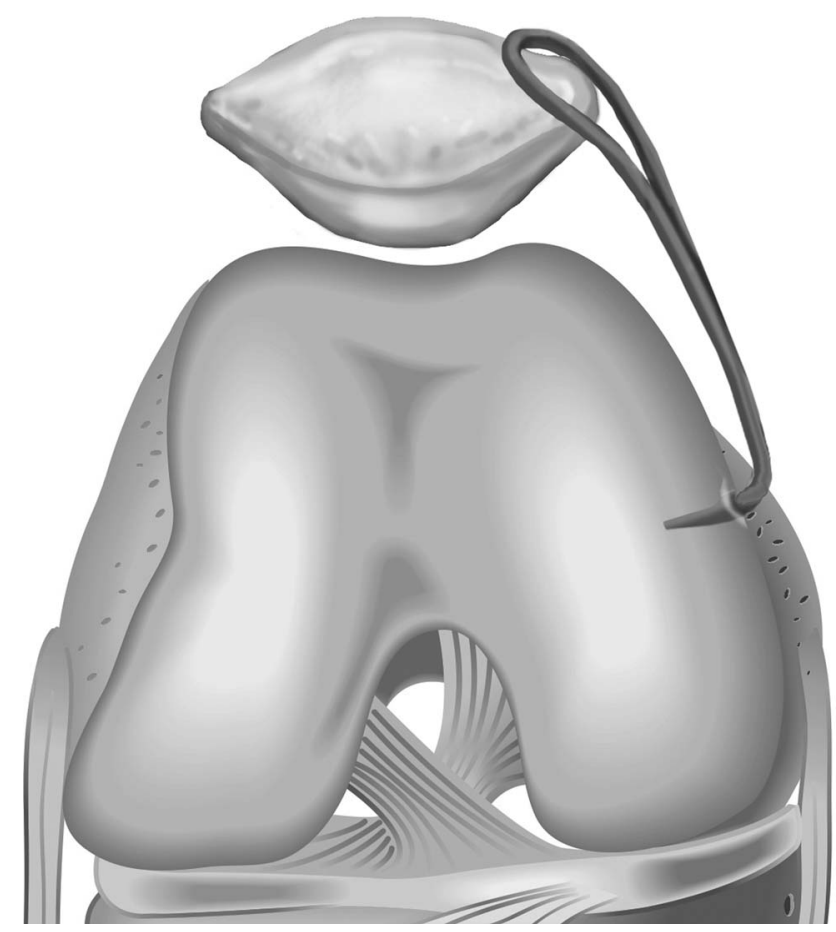

Figure 2. Schematic depicts the graft fixation technique with an L-shaped patellar tunnel and interference screw fixation in the femur.

tendon autograft. The autograft gracilis tendon was harvested after diagnostic arthroscopy and before proceeding with MPFL reconstruction via a previously described technique. ${ }^{18}$ Group 2 consisted of MPFL reconstructions using fresh-frozen gracilis tendon allograft obtained from the Musculoskeletal Transplant Foundation.

Group 1 patients underwent their index procedure between November 2012 and June 2013, and Group 2 patients underwent their MPFL reconstruction between November 2013 and January 2015. The change in technique occurred because of a concerning frequency of graft failures. Age at surgery, sex, left vs right knee, and chronicity of patellar instability were documented.

\section{Surgical Technique}

With the exception of graft harvest, the surgical techniques in both groups were identical. Standard diagnostic arthroscopy was performed using a 30-degree, 4-mm arthroscope (ConMed Linvatec) to determine any concomitant pathology. Secondary procedures were performed as indicated, including chondroplasty, loose body removal, microfracture, and meniscal repair using standard techniques. A superolateral arthroscopic portal was then made for viewing the patellofemoral tracking. Once patellar instability and maltracking were confirmed (Figure 1A), the decision to proceed with MPFL reconstruction was made. A small incision was made along the medial border of the patella. An L-shaped tunnel was created in the proximal third of the patella by drilling the medial and anterior surfaces with a 3.5-mm drill bit (Figure 2). Fluoroscopy was used to locate the Schöttle point on the femur, and a guidewire was drilled from medial to lateral out the lateral thigh. A reamer 
was used to overdrill the guidewire. A soft tissue tunnel was made between the MPFL insertion and medial patella tunnel, between the knee joint capsule and the vastus medialis obliquus. The graft was passed through the patellar tunnel using a Spectrum suture passer (ConMed Linvatec), and the free ends of the graft were sutured together using \#2 SutureLoop (Arthrex Inc). Using the SutureLoop, the graft was passed through the soft tissue tunnel and then through the distal femur. Appropriate patellar tracking was confirmed via arthroscopy. The knee was held in 30 degrees of flexion, and the graft was tensioned appropriately, so the patella sat in the center of the trochlear groove. A Matryx interference screw (ConMed Linvatec) was used to secure the graft. Final patellar tracking was confirmed with arthroscopy (Figure 1B).

\section{Clinical and Radiographic Assessment}

Clinical failure was defined as graft failure with recurrent instability and the need for revision MPFL reconstruction. The Kujala Scoring Questionnaire was chosen to assess clinical outcomes because it is a validated measure of clinical outcomes in patellofemoral conditions in this patient population. The Kujala Scoring Questionnaire assesses anterior knee pain on a scale of 1-100, with higher scores correlating with better function. ${ }^{22}$ Postoperative Kujala scores were collected $>6$ months after surgery when patients were considered fully recovered.

The TT-TG distance was measured on the preoperative magnetic resonance imaging (MRI) scan of each patient. The mean distance was computed and compared for patients who had MPFL reconstruction failures and for patients who had successful surgeries.

\section{Cost Analysis}

The financial department at our institution calculated procedure costs based on operative time differences between the autograft and allograft techniques. The cost of the allograft gracilis tendon was determined based on the national average obtained from the Musculoskeletal Transplant Foundation. The cost for reoperation was based on the procedure cost to the payer and to the hospital.

\section{Statistical Analysis}

All data were collected and compiled using Microsoft Excel (Microsoft Corporation). Statistical analyses were performed using SAS v.9.4 (SAS Institute Inc.). Chi-square and 2-sample $t$ test were respectively used to compare categorical and continuous variables between the groups. Wilcoxon 2-sample test was used when variables did not follow a normal distribution. A $P$ value of $<0.05$ indicates significance.

\section{RESULTS}

A total of 56 patients underwent MPFL reconstruction by the senior author from 2012-2015. Group 1 (gracilis autograft) contained 21 patients, while Group 2 (gracilis allograft) had 35 patients. Patient demographics are presented in Table 1. None of the demographic variables was statistically different between the groups. The average age in Group 1 was 15.3 years, while the average age of Group 2 patients was 16 years. Both groups had a female predominance, and the majority of patients in each group had chronic patellar instability. Allograft was used in 4 patients for revision MPFL reconstruction.
Table 1. Patient Demographics

\begin{tabular}{lccc}
\hline Variable & $\begin{array}{c}\text { Autograft } \\
\text { Group } \\
(\mathbf{n = 2 1 )}\end{array}$ & $\begin{array}{c}\text { Allograft } \\
\text { Group } \\
(\mathbf{n = 3 5 )}\end{array}$ & P Value \\
\hline Mean age, years & 15.3 & 16.0 & 0.45 \\
Males & $4(19.1)$ & $11(31.4)$ & 0.48 \\
Females & $17(80.9)$ & $24(68.6)$ & \\
Left knee & $14(66.7)$ & $20(57.1)$ & 0.67 \\
Right knee & $7(33.3)$ & $15(42.9)$ & \\
Acute patella dislocations & $5(23.8)$ & $12(34.3)$ & 0.59 \\
Chronic patella dislocations & $16(76.2)$ & $23(65.7)$ & \\
\hline
\end{tabular}

With the exception of age, data are reported as $n(\%)$.

Table 2. Autograft vs Allograft Outcomes

\begin{tabular}{|c|c|c|c|}
\hline Outcome & $\begin{array}{c}\text { Autograft } \\
\text { Group } \\
(n=21)\end{array}$ & $\begin{array}{l}\text { Allograft } \\
\text { Group } \\
(n=35)\end{array}$ & $P$ Value \\
\hline Graft failure, n (\%) & $6(28.6)$ & $0(0)$ & 0.0037 \\
\hline Average time to failure, months & 13.8 & $\mathrm{~N} / \mathrm{A}$ & $\mathrm{N} / \mathrm{A}$ \\
\hline $\begin{array}{l}\text { Average postoperative Kujala } \\
\text { score }^{a}\end{array}$ & 80.3 & 92.1 & 0.0032 \\
\hline Average graft size, $\mathrm{mm}$ & 5.29 & 5.7 & 0.0009 \\
\hline Average surgical time, minutes & 134.5 & 97.3 & 0.00002 \\
\hline
\end{tabular}

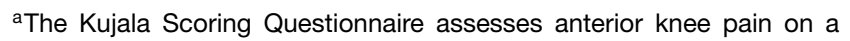
scale of 1-100, with higher scores correlating with better function. $\mathrm{N} / \mathrm{A}$, not applicable.

A total of 6 graft failures occurred, all in Group 1 and all in patients with chronic patellar instability (Table 2). This $28.6 \%$ failure rate prompted the change to using gracilis allograft tendons. The average time to graft failure was 13.8 months.

Patient clinical outcomes, assessed with Kujala scores, were significantly better in Group 2 . The graft was significantly larger in diameter in Group $2(5.29 \mathrm{~mm}$ vs $5.7 \mathrm{~mm}$, $P=0.0009$ ). Although this difference was statistically significant, it was not clinically significant as the current recommended graft diameter is $5-6 \mathrm{~mm} .{ }^{13}$ We found no difference in TT-TG distance in the grafts that failed vs those that were successful (Table 3).

Table 3. Comparison of Tibial Tubercle-Trochlear Groove (TT-TG) Distance in the Reconstruction Failures vs Reconstruction Successes Groups

\begin{tabular}{cccc}
\hline & $\begin{array}{c}\text { Reconstruction } \\
\text { Failures } \\
(\mathbf{n = 6 )}\end{array}$ & $\begin{array}{c}\text { Reconstruction } \\
\text { Successes } \\
(\mathbf{n = 5 0 )}\end{array}$ & P Value \\
\hline $\begin{array}{c}\text { Average TT-TG } \\
\text { distance, } \mathrm{mm}^{\mathrm{a}}\end{array}$ & 17.65 & 16.04 & 0.477 \\
\hline
\end{tabular}

aThe TT-TG distance was measured on the preoperative magnetic resonance imaging scan of each patient, and then the mean distance was calculated for each group. 
On average, the operating time was 37.2 minutes longer in Group 1, equating to a cost increase of approximately $\$ 445$. Based on information from the Musculoskeletal Transplant Foundation, each gracilis allograft was estimated to cost the patients in Group $2 \$ 1,058$. Reoperations were a significant financial burden, with $\$ 34,740$ in costs to the payer and approximately $\$ 4,500$ in costs to the hospital. After factoring in the costs of reoperation, allograft procedures were less expensive than autograft surgeries.

\section{DISCUSSION}

The MPFL is injured or torn in $>94 \%$ of patellar dislocations. ${ }^{7,8}$ Superior results in outcomes, complications, and failures have been reported with MPFL reconstruction vs conservative treatment. ${ }^{9,10,21,23}$ Our study of pediatric patients revealed superior graft survivorship in allograft MPFL reconstructions with $0 \%$ failures, which is significantly lower than the $28.6 \%$ failure seen in our autograft reconstructions.

In contrast to our results, Calvo Rodríguez et $\mathrm{al}^{24}$ retrospectively studied 13 autograft and 16 allograft MPFL reconstructions in adults and reported 0 recurrent dislocations or graft complications at a minimum 12-month follow-up. The difference in the results of our study compared to the Calvo Rodríguez et al study could be attributed to the shorter reported follow-up period in their study. The Calvo Rodríguez et al study only required 12 months of follow-up, whereas our study showed that graft failure occurred an average of 13.8 months after the procedure. In a study that included 25 MPFL reconstructions exclusively with gracilis allograft, Hohn and Pandya reported a $92 \%$ success rate with no episodes of instability at an average 24-month follow-up (range, 12-44 months). ${ }^{25}$ The low incidence of allograft failure during an extended follow-up period supports our findings of successful allograft gracilis MPFL reconstructions.

One reason for our failure rate in the autograft group could be the graft size. Our autograft patient population consisted entirely of pediatric patients with an average graft size of $5.29 \mathrm{~mm}$. Although no reported graft size is recommended for MPFL reconstructions, anterior cruciate ligament reconstructions were found to have failure rates 7 times higher when the graft was $<7 \mathrm{~mm}$ and lower failure rates with thicker grafts. ${ }^{26}$ To our knowledge, similar findings have not been reported with MPFL reconstructions. The native MPFL has an average midpoint width of $17.8 \mathrm{~mm}^{27}$; however, MPFL reconstructions have been described with average graft diameters of 5-6 mm without evidence of high failure rates. ${ }^{13}$

Another possible reason for the failure rates with autografts is underlying hypermobility and joint laxity. The link between joint laxity and patellar instability dates to 1958, when Carter and Sweetnam reported a family with a history of patellar dislocations and/or joint laxity. ${ }^{28}$ In 2016, Khemka et al published a retrospective review of 29 patients who underwent MPFL reconstruction using artificial ligaments and noted that 17 of the 29 patients had underlying ligamentous laxity. ${ }^{29}$ The Beighton score is a validated measure to assess for hypermobility in pediatric patients. ${ }^{30,31}$ Despite this suspected correlation, we did not routinely conduct Beighton scoring on our patients before this study was completed, and this focus could inform future studies.
We cannot exclude human or technical error as possible reasons for the increased success with allograft MPFL reconstructions. As previously stated, the senior surgeon performing the procedures initially used gracilis autografts for MPFL reconstructions but changed the technique to gracilis allograft after a pattern of autograft failures. Because the allograft reconstructions were done later, the surgeon's experience level could have affected the technical aspects of the reconstructions. Thus, the autograft failure rate could be partially attributed to the learning curve faced by the surgeon early in the series. He made no other changes to the technique.

To further investigate the high failure rate of autograft reconstructions, we measured the TT-TG distance on all preoperative MRIs and observed no difference in distance between the grafts that failed and those that were successful. Wagner et al prospectively examined risk factor effects on outcomes of MPFL reconstruction using gracilis autograft and reported that TT-TG distance $>20 \mathrm{~mm}$ was an indication for additional procedures, such as tibial tuberosity transfer. ${ }^{32}$ Although no tibial tuberosity transfers were performed on our patients, we found an overall trend toward larger TT-TG distances in our failures. Of the 6 failures, 2 (33.3\%) had TT-TG distances $>20 \mathrm{~mm}$ compared to $6(12.0 \%)$ of the 50 successful MPFL reconstructions. Even if the failures in these 2 patients were caused by a TT-TG $>20 \mathrm{~mm}$, more failures still occurred in the autograft group (4 vs 0 , respectively).

Our patients had overall good or fair functional outcomes as assessed by Kujala scores, although we observed significantly higher scores in our allograft group. To our knowledge, this is the first study to report such contrasting outcomes. Calvo Rodríguez et al also observed high Kujala scores in their allograft group compared to the autograft group (92.6 vs 89.2); however, the difference did not reach statistical significance. ${ }^{24}$ Several studies using only gracilis autograft for MPFL reconstructions have reported good outcomes and improvement in Kujala scores. ${ }^{32-34}$ The trend seen by Calvo Rodríguez et al, coupled with our findings, supports the use of gracilis allografts for MPFL reconstruction.

One of the biggest strengths of this study is the comparison of autograft gracilis to allograft gracilis tendon grafts for MPFL reconstruction. To our knowledge, our study is the first to compare these techniques in this age group, offering a direct comparison between autografts and allografts of the same tendon. Gracilis tendon was chosen because of the ease of harvest and the wide availability of allografts. Although the gracilis tendon is weaker than the oftenused semitendinosus tendon, it behaves more like the native MPFL. ${ }^{3,4,33}$ In a systemic review, Stupay et al demonstrated that semitendinosus grafts are more popular, having been used exclusively in 17 studies compared to exclusive gracilis use in 3 studies and mixed use in 11 studies. ${ }^{21}$ However, Stupay et al also noted that outcomes were similar between the semitendinosus and gracilis graft groups, with Kujala scores of 89.0 and 89.6 , respectively $(P=0.8) .{ }^{21}$ Additionally, autograft gracilis techniques have been described with overall satisfactory outcomes, ${ }^{32,34,35}$ which reassured us that our selection of gracilis tendon graft did not adversely affect our outcomes.

We found that autograft MPFL reconstructions were, on average, 37.2 minutes longer than allograft reconstructions. This time increase was expected given that the gracilis 
tendon needed to be harvested and the donor site closed in the autograft cohort. Another possible contribution to the additional operative time in the autograft group is the experience level of the senior surgeon. As noted previously, the autograft MPFL reconstructions were the procedure of choice at the beginning of the senior surgeon's career. When he performed the allograft reconstructions, the senior surgeon was more experienced.

The additional operative time for the autograft MPFL reconstructions was estimated to add $\$ 445$ in procedural costs compared to procedural costs of the allograft reconstructions. On the other hand, the MPFL reconstruction costs are increased by the cost of the allograft, which was estimated to be $\$ 1,058$. Consequently, the autograft MPFL reconstructions initially appeared to cost $\$ 613$ less than the allograph reconstructions. However, $28.6 \%$ of our autograft cohort underwent reoperation for MPFL reconstruction failure. To account for the higher failure and reoperation rates in the autograft cohort, we distributed the costs of reoperation ( $\$ 34,740$ to the patient/insurer and $\$ 4,500$ to our institution) among all autograft patients, resulting in more than $\$ 11,000$ ([cost savings of $\$ 34,740+\$ 4,500 \times 6$ failures] $\div$ 21 autograft patients) in additional costs per autograft MPFL reconstruction and making it the costlier procedure. With the increasing trend toward cost-effective healthcare, allograft MPFL reconstruction is the more economic option.

This study has several limitations. First, some performance bias could be present because the autograft reconstructions were done earlier in the series. Second, preoperative Kujala scores were not available, so we could not report overall improvement in our patients. Third, we had a relatively small sample size, but ours is currently the largest study directly comparing these 2 graft options. Large, randomized controlled studies would be ideal for conducting further direct comparisons between gracilis autograft and allograft MPFL reconstructions. Based on the current results, however, such studies might be hard to justify.

\section{CONCLUSION}

This study supports the use of gracilis allograft for MPFL reconstruction in skeletally mature pediatric patients. Gracilis allograft tendon had increased survivorship and superior Kujala scores compared to its autograft counterpart. The use of allograft tendon resulted in less operative time for the index procedure and a lower procedural cost when accounting for reoperation costs and rates seen with the use of gracilis autograft tendon.

\section{ACKNOWLEDGMENTS}

The authors have no financial or proprietary interest in the subject matter of this article. This work has been presented at the 2016 Arthroscopy Association of North America Annual Meeting, April 14-16, 2016, Boston, MA.

\section{REFERENCES}

1. Nietosvaara Y, Aalto K, Kallio PE. Acute patellar dislocation in children: incidence and associated osteochondral fractures. J Pediatr Orthop. 1994 Jul-Aug;14(4):513-515.

2. Fithian DC, Paxton EW, Stone ML, et al. Epidemiology and natural history of acute patellar dislocation. Am J Sports Med. 2004 Jul-Aug;32(5):1114-1121.

doi: $10.1177 / 0363546503260788$.
3. Steensen RN, Dopirak RM, McDonald WG 3rd. The anatomy and isometry of the medial patellofemoral ligament: implications for reconstruction. Am J Sports Med. 2004 Sep;32(6):1509-1513. doi: 10.1177/0363546503261505.

4. Shea KG, Polousky JD, Jacobs JC, et al. The patellar insertion of the medial patellofemoral ligament in children: a cadaveric study. J Pediatr Orthop. 2015 Jun;35(4):e31-e35. doi: $10.1097 /$ BPO.0000000000000399.

5. Conlan T, Garth WP Jr, Lemons JE. Evaluation of the medial soft-tissue restraints of the extensor mechanism of the knee. $J$ Bone Joint Surg Am. 1993 May;75(5):682-693.

6. Desio SM, Burks RT, Bachus KN. Soft tissue restraints to lateral patellar translation in the human knee. Am J Sports Med. 1998 Jan-Feb;26(1):59-65. doi: 10.1177/03635465980260012701.

7. Sallay PI, Poggi J, Speer KP, Garrett WE. Acute dislocation of the patella. A correlative pathoanatomic study. Am J Sports Med. 1996 Jan-Feb;24(1):52-60. doi: 10.1177/036354659602400110.

8. Sanders TG, Morrison WB, Singleton BA, Miller MD, Cornum KG. Medial patellofemoral ligament injury following acute transient dislocation of the patella: MR findings with surgical correlation in 14 patients. J Comput Assist Tomogr. 2001 Nov-Dec;25(6):957-962.

9. Schneider DK, Grawe B, Magnussen RA, et al. Outcomes after isolated medial patellofemoral ligament reconstruction for the treatment of recurrent lateral patellar dislocations: a systematic review and meta-analysis. Am J Sports Med. 2016 Nov;44(11):2993-3005. doi: 10.1177/0363546515624673.

10. Bitar AC, Demange MK, D'Elia CO, Camanho GL. Traumatic patellar dislocation: nonoperative treatment compared with MPFL reconstruction using patellar tendon. Am J Sports Med. 2012 Jan;40(1):114-122. doi: 10.1177/0363546511423742.

11. Russ SD, Tompkins M, Nuckley D, Macalena J. Biomechanical comparison of patellar fixation techniques in medial patellofemoral ligament reconstruction. Am J Sports Med. 2015 Jan;43(1):195-199. doi: 10.1177/0363546514550992.

12. Ahmad R, Jayasekera N, Schranz P, Mandalia V. Medial patellofemoral ligament reconstruction: a technique with a "v"-shaped patellar tunnel. Arthrosc Tech. $2014 \mathrm{Sep}$ 22;3(5):e589-e592. doi: 10.1016/j.eats.2014.06.015.

13. Ahmad CS, Brown GD, Shubin Stein BE. The docking technique for medial patellofemoral ligament reconstruction: surgical technique and clinical outcome. Am J Sports Med. 2009 Oct;37(10):2021-2027. doi: 10.1177/0363546509336261.

14. Kepler CK, Bogner EA, Hammoud S, Malcolmson G, Potter HG, Green DW. Zone of injury of the medial patellofemoral ligament after acute patellar dislocation in children and adolescents. Am J Sports Med. 2011 Jul;39(7):1444-1449. doi: $10.1177 / 0363546510397174$.

15. Haupert A, Lorbach O. Anatomic reconstruction of the medial patellofemoral ligament using the fascia lata as an autograft. Arthrosc Tech. 2015 Feb 9;4(1):e57-e63.

doi: 10.1016/j.eats.2014.11.005.

16. Lorbach O, Haupert A, Efe T, et al. Biomechanical evaluation of MPFL reconstructions: differences in dynamic contact pressure between gracilis and fascia lata graft. Knee Surg Sports Traumatol Arthrosc. 2017 Aug;25(8):2502-2510. doi: 10.1007/s00167-016-4005-5.

17. Nakagawa S, Arai Y, Kan H, et al. Medial patellofemoral ligament reconstruction procedure using a suspensory femoral fixation system. Arthrosc Tech. 2013 Nov 15;2(4):e491-e495. doi: 10.1016/j.eats.2013.07.009.

18. Kim TS, Kim HJ, Ra IH, Kyung HS. Medial patellofemoral ligament reconstruction for recurrent patellar instability using a gracilis autograft without bone tunnel. Clin Orthop Surg. 2015 Dec;7(4):457-464. doi: 10.4055/cios.2015.7.4.457. 
19. Kang H, Cao J, Yu D, Zheng Z, Wang F. Comparison of 2 different techniques for anatomic reconstruction of the medial patellofemoral ligament: a prospective randomized study. Am J Sports Med. 2013 May;41(5):1013-1021. doi: $10.1177 / 0363546513480468$.

20. Shah JN, Howard JS, Flanigan DC, Brophy RH, Carey JL, Lattermann C. A systematic review of complications and failures associated with medial patellofemoral ligament reconstruction for recurrent patellar dislocation. Am J Sports Med. 2012 Aug;40(8):1916-1923. doi: $10.1177 / 0363546512442330$.

21. Stupay KL, Swart E, Shubin Stein BE. Widespread implementation of medial patellofemoral ligament reconstruction for recurrent patellar instability maintains functional outcomes at midterm to long-term follow-up while decreasing complication rates: a systematic review. Arthroscopy. 2015 Jul;31(7):1372-1380. doi: 10.1016/j.arthro.2014.12.029.

22. Kujala UM, Jaakkola LH, Koskinen SK, Taimela S, Hurme M, Nelimarkka O. Scoring of patellofemoral disorders. Arthroscopy. 1993;9(2):159-163.

23. Mackay ND, Smith NA, Parsons N, Spalding T, Thompson P, Sprowson AP. Medial patellofemoral ligament reconstruction for patellar dislocation: a systematic review. Orthop J Sports Med. 2014 Aug 8;2(8):2325967114544021. doi: $10.1177 / 2325967114544021$.

24. Calvo Rodríguez R, Figueroa Poblete D, Anastasiadis Le Roy Z, Etchegaray Bascur F, Vaisman Burucker A, Calvo Mena R. Reconstruction of the medial patellofemoral ligament: evaluation of the clinical results of autografts versus allografts [in English, Spanish]. Rev Esp Cir Ortop Traumatol. 2015 Sep-Oct;59(5):348-353. doi: 10.1016/j.recot.2014.10.006.

25. Hohn E, Pandya NK. Does the utilization of allograft tissue in medial patellofemoral ligament reconstruction in pediatric and adolescent patients restore patellar stability? Clin Orthop Relat Res. 2017 Jun;475(6):1563-1569. doi: 10.1007/s11999-016-5060-4.

26. Magnussen RA, Lawrence JT, West RL, Toth AP, Taylor DC, Garrett WE. Graft size and patient age are predictors of early revision after anterior cruciate ligament reconstruction with hamstring autograft. Arthroscopy. 2012 Apr;28(4):526-531. doi: 10.1016/j.arthro.2011.11.024.

27. Kyung HS, Kim HJ. Medial patellofemoral ligament reconstruction: a comprehensive review. Knee Surg Relat Res. 2015 Sep;27(3):133-140. doi: 10.5792/ksrr.2015.27.3.133.

28. Carter C, Sweetnam R. Familial joint laxity and recurrent dislocation of the patella. J Bone Joint Surg Br. 1958 Nov;40-B(4):664-667.

29. Khemka A, Lord SJ, Doyle Z, Bosley B, Al Muderis M. Minimally invasive medial patellofemoral ligament reconstruction for patellar instability using an artificial ligament: a two year follow-up. Knee. 2016 Mar;23(2):261-266. doi: 10.1016/j.knee.2015.07.002.

30. Beighton $\mathrm{P}$, Solomon L, Soskolne CL. Articular mobility in an African population. Ann Rheum Dis. 1973 Sep;32(5): 413-418.

31. Smits-Engelsman B, Klerks M, Kirby A. Beighton score: a valid measure for generalized hypermobility in children. $J$ Pediatr. 2011 Jan;158(1):119-123, 123.e1-e4. doi: 10.1016/j.jpeds.2010.07.021.

32. Wagner D, Pfalzer F, Hingelbaum S, Huth J, Mauch F, Bauer G. The influence of risk factors on clinical outcomes following anatomical medial patellofemoral ligament (MPFL) reconstruction using the gracilis tendon. Knee Surg Sports Traumatol Arthrosc. 2013 Feb;21(2):318-324. doi: 10.1007/s00167-012-2015-5.

33. Amis AA, Firer P, Mountney J, Senavongse W, Thomas NP. Anatomy and biomechanics of the medial patellofemoral ligament. Knee. 2003 Sep;10(3):215-220.

34. Schottle PB, Romero J, Schmeling A, Weiler A. Technical note: anatomical reconstruction of the medial patellofemoral ligament using a free gracilis autograft. Arch Orthop Trauma Surg. 2008 May; 128(5):479-484. doi: 10.1007/s00402-007-0300-4.

35. Christiansen SE, Jacobsen BW, Lund B, Lind M. Reconstruction of the medial patellofemoral ligament with gracilis tendon autograft in transverse patellar drill holes. Arthroscopy. 2008 Jan;24(1):82-87. doi: 10.1016/j.arthro.2007.08.005.

This article meets the Accreditation Council for Graduate Medical Education and the American Board of Medical Specialties Maintenance of Certification competencies for Patient Care, Medical Knowledge, and Practice-Based Learning and Improvement. 